Meta

Journal des traducteurs

Translators' Journal

\title{
Fonctions et activités de l'Académie de la langue hébraïque dans l'orientation et le développement de la langue hébraïque
}

\section{Moché Bar-Acher}

Volume 43, numéro 1, mars 1998

La traduction et l'interprétation en Israël

Translation and Interpreting in Israel

URI : https://id.erudit.org/iderudit/001967ar

DOI : https://doi.org/10.7202/001967ar

Aller au sommaire du numéro

Éditeur(s)

Les Presses de l'Université de Montréal

ISSN

0026-0452 (imprimé)

1492-1421 (numérique)

Découvrir la revue

Citer cet article

Bar-Acher, M. (1998). Fonctions et activités de l'Académie de la langue hébraïque dans l'orientation et le développement de la langue hébraïque. Meta, 43(1), 10-18. https://doi.org/10.7202/001967ar
Résumé de l'article

Cet article retrace les principales étapes de la renaissance de la langue hébraïque et de son utilisation comme langue moderne à travers les efforts des membres du Comité de la langue hébraïque (Jérusalem, 1889-1953), bientôt relayé, après la création de l'État d'Israël en mai 1948, par l'Académie de la langue hébraïque, établie en 1953 par décision de la Knes- seth (le Parlement israélien). L'auteur expose les objectifs et les principales fonctions de l'Académie telles qu'elles sont définies dans ses statuts. Il décrit les activités de recherche de l'Académie et dépeint ses relations avec d'autres institutions nationales. 


\title{
FONCTIONS ET ACTIIVITÉS DE L'ACADÉMIE DE LA LANGUE HÉBRAIIQUE DANS L'ORIENTATION ET LE DEVELOPPEMENT DE LA LANGUE HÉBRAIIQUE
}

\author{
MOCHÉ BAR-ACHER \\ Président de l'Académie de la langue hébraïque et \\ Université hébräque de Jérusalem, Jérusalem, Israël
}

\begin{abstract}
Résumé
Cet article retrace les principales étapes de la renaissance de la langue hébräque et de son utilisation comme langue moderne à travers les efforts des membres du Comité de la langue hébraïque (Jérusalem, 1889-1953), bientôt relayé, après la création de l'État d'Israël en mai 1948, par l'Académie de la langue hébraïque, établie en 1953 par décision de la Knesseth (le Parlement israélien). L'auteur expose les objectifs et les principales fonctions de l'Académie telles qu'elles sont définies dans ses statuts. Il décrit les activités de recherche de l'Académie et dépeint ses relations avec d'autres institutions nationales.
\end{abstract}

\begin{abstract}
This article examines the principal stages of the rebirth of Hebrew and its use as a modern language through the efforts of the Hebrew Language Committee (Jerusalem, 18891953) and pursued, after the creation of the State of Israel in May 1948, by the Hebrew Language Academy established by the Israeli Parliament, the Knesseth. The author outlines the objectives and main functions of the Academy as defined in its statutes and describes its research activities and relations with other national institutions.
\end{abstract}

\section{INTRODUCTION : LA RENAISSANCE DE L'HÉBREU ET LE COMITÉ de la laNgUe HÉBRAÏQUE}

Lorsqu'un pays fonde une Académie de la langue, il possède généralement une langue locale bien vivante. C'est ainsi que l'Académie française fut fondée à une époque où le français était parlé et écrit tant par le petit peuple que par les écrivains et les lettrés.

Ce qui apparaît un peu partout comme une évidence n'alla pas de soi en ce qui concerne l'Académie de la langue hébraïque ou l'institution qui l'avait précédée, en Palestine, le Comité de la langue hébraïque. Lorsque ce dernier se constitua, en 1889, à Jérusalem, l'hébreu n'était pas la langue vernaculaire des Juifs de Palestine ni des Juifs de l'étranger. Les Juifs de Palestine s'exprimaient dans des langues diverses, tel l'arabe (la langue de la population locale) ou dans d'autres langues qu'ils avaient rapportées de leurs pays d'origine. Certaines étaient des langues «juives», comme le parler des Juifs d'Europe : le yiddish, ou comme le judéo-espagnol employé par les Juifs des Balkans tandis que les Juifs d'Afrique du Nord utilisaient l'arabe judéo-maghrébin. D'autres étaient les langues de l'Europe moderne : le français, l'allemand, etc.

Quant à l'hébreu, il avait cessé d'être parlé près de 1800 ans plus tôt, quelques décennies après la chute de l'État juif et la destruction du Second Temple, subsistant uniquement comme langue de culture : dans le rite synagogal et en tant que langue écrite (au début exclusivement pour l'usage religieux puis pour tout autre usage). En d'autres termes, l'hébreu

Meta, XLIII, 1, 1998 
ne devint jamais vraiment une langue morte. S'il cessa d'être une langue parlée, il resta une langue savante, la langue de la culture juive. Désormais, les Juifs allaient vivre en situation de diglossie : écrivant une langue et en parlant une autre. Ce bilinguisme explique que de nombreux vocables et des expressions empruntés aux textes sacrés et rabbiniques pénétrèrent dans les judéo-langues parlées par les Juifs.

À la fin du XIX ${ }^{\mathrm{e}}$ siècle, avec la réapparition d'une identité politique du peuple juif, on éprouva le besoin de redonner à l'hébreu son statut de langue parlée. Il fallait tout d'abord, pour des raisons sociales et humaines, fournir une langue commune aux Juifs immigrés de nombreux pays qui parlaient des langues diverses. Le fait que les érudits, religieux et laïques, aient connu et utilisé l'hébreu littéraire constitua un point de départ important. Bien entendu, cette renaissance linguistique n'était pas dépourvue de motivations idéologiques. Comment imaginer un renouveau juif politique sans renaissance de l'hébreu comme langue parlée, langue vivante sous tous ses aspects ? C'est dans cette conjoncture que naquit le Comité de la langue hébraïque.

Le Comité fut créé à l'initiative d'Eliézer Ben-Yehouda (connu comme le «Père de la renaissance de l'hébreu»), entouré de trois autres lettrés. Après moins d'une année d'activités, sans résultats véritables, le Comité interrompit ses travaux. Reconstitué en 1904, il fonctionna alors jusqu'en 1953. Cette seconde période fut d'autant plus féconde que le Comité s'était assuré le soutien de l'Association des enseignants. La fonction essentielle du Comité consista à fournir les mots de l'école et de l'usage quotidien et à faire en sorte que tous les lycées et toutes les localités de Palestine utilisent simultanément le même vocable. Par exemple, lorsque les néologismes cha'one («montre») et 'iparone («crayon») furent créés, il devint essentiel que tout le monde emploie le même mot, à travers tout le pays, et non pas que des néologismes différents soient introduits parallèlement, dans telle ou telle école. Le fait que David Yelin, l'un des piliers du Comité, ait été en même temps le Président de l'Association des enseignants et qu'il ait fondé une École normale (de formation des maîtres) favorisa la réussite de l'entreprise. (Il faut néanmoins souligner qu'on ne peut attribuer tout le mérite de la renaissance de la langue hébraïque au Comité ou aux enseignants. Il existait d'autres moteurs de cette renaissance linguistique, très actifs essentiellement à Tel-Aviv, la première ville juive, créée en 1909. Mais cela n'enlève rien au mérite des deux institutions citées, dont l'importance est reconnue de tous.)

Le rôle essentiel du Comité fut donc de fournir les mots de la vie courante. Mais d'autres besoins et d'autres problèmes surgirent, comme la question de la prononciation officielle de l'hébreu palestinien. En effet, chaque communauté d'origine avait emporté avec elle ses habitudes, son accent. Certains traits anciens s'étaient conservés dans les différentes traditions juives, mais des traits nouveaux étaient apparus, empruntés aux diverses langues du monde. C'est ainsi que le nom de la ville de Jérusalem pouvait être prononcé de plusieurs manières : Yerouchalayim, Iroucholaym, Iroussalaym, Yaroucholayim, etc. Il fallait donc que le Comité prenne aussi position sur ce point. On décida d'adopter l'accent conservé dans le parler hébreu des Juifs d'Orient. Cet «accent oriental» était le plus proche de l'accent antique à plus d'un égard. C'était du moins l'avis de la majorité des spécialistes du Comité, ainsi que des experts d'hébreu et de langues sémitiques consultés par le Comité dans les universités d'Europe. Notons pourtant que sur cette question, et sur bien d'autres d'ailleurs, le Comité dut faire des compromis. Ayant décidé d'adopter la prononciation sefardi (orientale), il admit néanmoins quelques-uns des principes de la prononciation achkenazi (européenne), en raison de la proportion importante des locuteurs originaires d'Europe, dans la population juive de Palestine et de la Diaspora. Par ailleurs, de nombreux usages en matière de prononciation s'imposèrent par le bas, c'est-à-dire qu'ils furent introduits par les locuteurs eux-mêmes, en contradiction avec les décisions du Comité de la langue (phénomène qu'on retrouve pour l'adoption de nombreux néologismes). 


\section{L'ACADÉMIE DE LA LANGUE HÉBRAIQUE}

\subsection{Fondation de l'Académie}

Lors de la création de l'État d'Israël, en 1948, les responsables du Comité, dirigés et encouragés par le premier Président du Conseil du pays, David Ben-Gourion, œuvrèrent pour la constitution d'une académie de la langue. C'était déjà le vœu des pionniers du Comité, dont les membres les plus éminents, tel Eliézer Ben-Yehouda (mentionné plus haut), puis, plus tard, 'Haïm Na'hman Bialik, surnommé «le Poète national», pensaient qu'il importait d'établir un institut qui fonderait son travail (d'orientation de la langue) sur l'étude de l'hébreu à toutes les époques. De plus, le Comité étant une organisation bénévole, l'adoption de ses recommandations dépendait du bon vouloir des institutions ou des particuliers. Il fallait créer une instance ayant autorité officielle et légale, ancrée dans la législation. C'est pourquoi, en 1953, le Parlement israélien (la Knesseth) vota une loi portant sur la création d'une Académie de la langue hébraïque, mettant fin par là même à l'existence du Comité de la langue hébraïque.

\subsection{Objectifs et fonctions de l'Académie}

Voici les objectifs de l'Académie tels qu'on les trouve formulés dans le premier chapitre de ses statuts :

a) Euvrer pour recueillir le patrimoine de la langue hébraïque de toutes les époques et de tous les niveaux de langue.

b) Effectuer des recherches sur la structure de l'hébreu, son histoire et ses transformations.

c) Orienter les modes de développement de la langue hébraïque, conformément à sa nature, à ses besoins, à ses possibilités, dans toutes les disciplines théoriques et pratiques, en ce qui concerne le vocabulaire, la grammaire, l'écriture, l'orthographe et les règles de transcription.

Rappelons pour mémoire qu'un quatrième objectif avait été défini à l'origine : «contribuer à la diffusion de la connaissance de la langue hébraïque». Cet objectif, qui aurait transformé la nature de l'Académie (d'institution législative en institution exécutive), fut finalement dévolu au ministère de l'Éducation et de la Culture et aux médias publics. Vérifions, à présent, dans quelle mesure ces objectifs et ces fonctions se sont traduits dans les faits. Nous passerons rapidement sur les deux premiers points et nous nous étendrons plus longuement sur le troisième.

\subsubsection{La collecte du patrimoine de la langue hébraïque}

L'Académie, dès sa constitution, s'attacha à collecter le vocabulaire hébraïque de toutes les époques. Pour ce faire, elle établit un programme de Dictionnaire historique de la langue hébrä̈que (cf., ci-après, l'article de Israël Yevin). Cette entreprise fut dirigée par l'un des plus éminents spécialistes contemporains de l'hébreu, le professeur Zeev Ben'Haïm, qui devint le second Président de l'Académie. On renonça, à ce stade, à traiter de la langue biblique, puisqu'il existait d'ores et déjà des concordances et de bons dictionnaires. On concentra les efforts sur les premières périodes postbibliques. La première tranche porta sur cinq siècles (du second siècle avant l'ère vulgaire jusqu'au troisième siècle après). Une seconde tranche couvrit les années 300 à 1050, et tous les documents écrits de cette période furent traités et inclus dans la banque de données du dictionnaire. On aborda, ensuite, une tranche plus «moderne», allant de 1750 à l'année 1920, réunissant des échantillons de plus en plus étoffés et nombreux. Nous possédons actuellement plusieurs dizaines de volumes de concordances détaillées faisant référence à tous les textes traités pour 
les périodes précitées. À présent, nous nous engageons dans un travail intensif portant sur certains blocs d'écrits appartenant à la tranche 1050-1750. Il faut préciser que l'intégralité du matériel est accessible à tout un chacun. Nul besoin d'appartenir à l'Académie ou d'habiter Israël. Chacun peut l'étudier et s'en servir. Le matériel de la première tranche (de - 200 à +300 ) a déjà été publié sur microfiches. Des centaines d'institutions et de particuliers les ont acquises et en font usage. Ceci sert le second objectif de l'Académie : la recherche.

\subsubsection{La recherche linguistique}

Il va sans dire que la recherche portant sur l'hébreu ne s'est pas confinée aux seuls murs de l'Académie de la langue hébraïque. Elle se poursuit également dans les universités d'Israël et du monde entier. Pourtant, depuis que l'Académie a entrepris de recueillir les textes hébreux et de les analyser, et depuis qu'elle a mis ce matériel à la disposition du public, des dizaines et des dizaines de chercheurs, au moins, ont appuyé leurs travaux sur la base de données de l'Académie. Nombreux sont les sujets de grammaire et de lexicologie hébraïques qui sont présentés aujourd'hui sous un jour radicalement différent, grâce aux données précises fournies à la recherche par le programme du dictionnaire historique. Le travail de collecte terminologique et les recherches qui en découlent constituent un maillon important du troisième volet des responsabilités de l'Académie : l'orientation du développement de la langue hébraïque.

\subsubsection{L'orientation de la langue}

Aux termes de ses statuts, l'Académie doit «orienter les modes de développement de la langue hébraïque [...] en ce qui concerne le vocabulaire, la grammaire, l'écriture, l'orthographe et les règles de transcription». C'est pourquoi, aussitôt après sa création, elle a mis en place des commissions chargées de réaliser ces objectifs.

\subsubsection{Les néologismes}

Comme cela avait été le cas à l'époque du Comité de la langue, l'Académie, au cours de ses quarante-cinq années d'existence, a constitué plusieurs dizaines de commissions spécialisées dans divers domaines pour fixer la terminologie. Nous citerons, à titre d'exemple, des commissions en médecine, technologie, sciences sociales, droit, botanique, etc., ainsi qu'une commission de vocabulaire général. Chaque commission est composée de membres de l'Académie et de spécialistes appartenant au domaine considéré. Une fois le travail de la commission terminé, le matériel réuni est envoyé pour relecture à de nombreux spécialistes ainsi qu'à l'ensemble des membres de l'Académie. Au vu des remarques, la commission complète ses travaux et transmet le glossaire à la plénière de l'Académie pour approbation. En général, la plénière se contente d'un débat sur un petit nombre de termes controversés. Une fois approuvé par l'Académie, le glossaire est avalisé par la signature du ministre de l'Éducation. Il est alors publié dans Rechoumoth, le Journal officiel de l'État d'Israël, avant d'être diffusé dans le grand public. L'Académie vient de réunir l'ensemble de la terminologie normalisée par le Comité de la langue et par elle-même. Il s'agit de plus de cent mille entrées. Peut-être convient-il de préciser que lorsque nous parlons de termes normalisés et de néologismes, nous ne retenons pas seulement les mots nouveaux, qui n'ont jamais été en usage dans la langue hébraïque. Nous incluons dans cette terminologie de nombreux vocables hébraïques utilisés à des époques antérieures avec une acception différente, auxquels une acception nouvelle a été attribuée dans notre terminologie. C'est ainsi qu'en hébreu biblique, le terme pidyiome est une variante libre du mot pidyione (rachat, rançon). L'Académie a réservé pidyiome à la terminologie bancaire, en lui donnant le sens de l'anglais on call (call money : emprunt, argent remboursable sur 
demande, argent au jour le jour). Cette acception a été favorisée par le fait qu'on pouvait considérer pidyiome comme un mot-valise composé des deux mots, pidyione et yom (jour). Cette démarche va dans le sens de la remarque exprimée par l'un des premiers responsables du Comité de la langue : «Ce qui est nouveau, dans les néologismes, c'est qu'ils ne sont pas nouveaux.» L'Académie inclut également dans ses listes des mots empruntés aux langues étrangères. C'est particulièrement fréquent dans les «langues de spécialité» qui, plus exclusives, créent un langage commun entre des professionnels de pays différents et de cultures variées. Mais l'Académie n'hésite pas, non plus, à introduire des emprunts dans le vocabulaire dit «général», lorsque les termes se sont imposés de manière internationale. Prenons l'exemple du mot téléphone. La famille Ben-Yehouda avait proposé un néologisme hébraïque : $s a^{\prime} h \mathrm{ra}^{\prime} h o k$, sorte de calque plus ou moins exact du mot téléphone ( $s a^{\prime} h / \ll$ conversation», $r a^{\prime} h o k /$ «à distance»). Néanmoins l'Académie a estimé qu'il fallait conserver le terme international, dont elle a dérivé un verbe : letalfène/téléphoner. Tout naturellement, la rue a développé des substantifs : talpane et talpanite («téléphoniste», au masculin et au féminin), ce qui prouve bien que le terme a clairement subi un processus d'assimilation à l'intérieur même de la langue hébraïque. L'Académie n'a pas jugé bon non plus d'adopter un vocable hébraïque pour se désigner elle-même. Elle s'appelle donc, en hébreu : académia, de la même manière que dans l'Antiquité, l'institution juive la plus importante de l'époque du Second Temple portait, en hébreu, un nom grec : le Sanhédrin.

Il importe de signaler deux points concernant l'activité terminologique de l'Académie :

1) Les néologismes proposés par l'Académie ne sont pas tous acceptés par la langue vivante. C'est ainsi que, pour traduire le mot «application», l'Académie avait dérivé un substantif d'un verbe existant pour créer hè'hala. Mais le public employait déjà un autre terme : yissoum, que l'Académie considérait comme grammaticalement incorrect et peu heureux. C'est pourtant yissoum qui l'a emporté et l'Académie a donc accepté le verdict et validé le mot. Mais bien entendu, de très nombreux termes ont été assimilés, tant dans la langue de tous les jours que dans les langues de spécialité, et sont couramment utilisés. C'est ainsi que le néologisme proposé pour désigner un répondeur téléphonique, mèchivone (construit sur le verbe lehachiv/«répondre») s'est imposé très rapidement. Le terme 'itsoumim («grèves perlées, de représailles») s'est imposé plus rapidement encore, supplantant le vocable international qui s'employait auparavant (sanktsioth/«sanctions»). Le processus d'adoption d'un terme par le public dépend de bien des facteurs, en premier lieu des médias. Le fait que les chaînes nationales de radio et de télévision emploient des conseillers linguistiques de l'Académie joue un rôle non négligeable. De nombreux néologismes sont introduits par l'intermédiaire de ces conseillers.

2) Il faut souligner, en second lieu, que la création de néologismes n'est pas une chasse gardée de l'Académie. Des écrivains, des journalistes, des militaires, des membres de professions libérales et d'autres institutions proposent de nouveaux mots. Ils le font parfois avant même l'Académie et leurs propositions sont admises, en fin de compte. C'est ainsi que pour traduire le concept américain de hit (qui désigne une chanson très populaire, aimée et qu'on entend partout), c'est un journaliste qui a eu l'idée d'employer un mot hébraïque dont les sonorités sont proches de l'américain : lahit, forgé sur une racine hébraïque très ancienne. Le mot a été préféré par l'Académie à d'autres propositions de ses propres membres. 


\subsubsection{Les questions de grammaire}

L'Académie est très active, également, dans le domaine de la grammaire et dans d'autres secteurs déjà évoqués. Pour les puristes, la grammaire hébraïque qui sert de modèle et de référence est la grammaire de l'hébreu biblique, dans la tradition de lecture qu'on appelle «tradition de Tibériade». Mise par écrit il y a plus de mille deux cents ans, elle s'est imposée peu à peu dans l'ensemble des communautés juives comme référence normative. Rien d'étonnant, donc, à ce que pratiquement toute l'activité philologique hébraïque, au cours des siècles, se soit appuyée sur la description de cette tradition. À l'époque de la renaissance de l'hébreu, c'est encore cette tradition qu'on a prise pour modèle et qu'on a décidé de suivre. L'Académie elle aussi considère que la grammaire biblique fonde la grammaire normative. Mais la science moderne a mis au jour d'autres traditions, grâce à l'étude de documents écrits ou de témoignages oraux recueillis de la bouche de juifs issus de communautés diverses. Si bien que l'Académie, dans ses décisions, tient compte aussi de ces autres traditions linguistiques. Mais par-dessus tout, l'Académie se veut attentive aux transformations naturelles qui ont eu lieu (et continuent d'avoir lieu) dans la langue vivante depuis plusieurs générations. N'oublions pas qu'il y a quatrevingts ans, seuls quelques dizaines d'enfants parlaient l'hébreu comme langue maternelle. Aujourd'hui, il y a bien plus de deux millions d'Israéliens pour qui l'hébreu est soit la langue maternelle, soit la langue dans laquelle ils ont été éduqués depuis leur petite enfance. Bien des changements se sont produits dans la grammaire de la langue vivante, à la suite de processus philologiques naturels, par exemple : l'analogie qui fait modifier des règles de grammaire, ou la différenciation sémantique. C'est ainsi que lorsque deux variantes grammaticales dotées de la même acception coexistent dans la grammaire normative, la langue vivante essaie souvent d'utiliser chacune avec une acception distincte. Prenons l'exemple de mazkira et mazkérète, qui sont deux variantes du participe présent au féminin du verbe lehazkir («rappeler», «évoquer»). À l'origine, ces deux variantes libres étaient rigoureusement équivalentes. Mais l'hébreu moderne les utilise également comme substantifs. Il les a donc distinguées en créant deux noms : mazkira («secrétaire», «employée de bureau») et mazkérète («cadeau-souvenir»). On pourrait évoquer encore de nombreux processus d'évolution de la langue vivante, dont l'Académie tient compte dans ses décisions. Soulignons également qu'il se trouve aujourd'hui quelques philologues, suivis par quelques journalistes, qui prônent une attitude laxiste dans la détermination des normes linguistiques, ce qui conduirait à s'éloigner nettement de la langue classique. L'Académie n'est pas favorable à une telle attitude, mais ne rejette pas pour autant la légitimité de la langue parlée, avec toutes ses caractéristiques. Elle considère qu'il convient aussi d'étudier et d'examiner, sur un plan philologique et stylistique, l'argot israélien, les déformations morphologiques, réelles ou supposées, le relâchement de la diction (qui perturbe parfois la communication) et d'autres formes qui caractérisent la langue parlée. Mais l'Académie considère qu'il faut être vigilant pour préserver la langue officielle et formelle. C'est à cette défense de la langue hébraïque qu'elle se consacre dans ses décisions fixant des normes de langage (sans parler d'autres tâches, sur lesquelles nous n'aurons pas le loisir de nous étendre ici).

\subsubsection{L'hébreu et l'araméen}

Il est temps d'évoquer le rôle important joué par l'araméen (langue sœur de l'hébreu) dans l'Antiquité et, dans une moindre mesure, à l'époque moderne. On retrouve une influence ininterrompue de l'araméen durant deux mille cinq cents ans, tant sur le vocabulaire que sur la grammaire hébraïque. À certaines époques, et dans certains textes, on peut parler de véritable symbiose entre les deux langues. La présence de l'araméen dans le texte hébreu est marquante. Il existe même des œuvres importantes dans lesquelles les deux lan- 
gues se suivent, alternent ou s'imbriquent totalement. Rien d'étonnant donc si, à l'époque de la renaissance hébraïque et dans son sillage, l'araméen servit de réservoir inépuisable à l'hébreu. Contentons-nous d'un seul exemple, parmi bien d'autres. Il existe, dans l'hébreu d'aujourd'hui, de très nombreux «doublets». (On sait que les doublets sont des paires de mots issus de racines similaires dans deux langues de la même famille qui coexistent dans une même langue.) L'hébreu moderne, surtout au moment de la renaissance linguistique et dans la période qui a suivi, présentait de très nombreux doublets, l'un des synonymes étant d'origine hébraïque, l'autre d'origine araméenne. Aujourd'hui, dans la plupart des cas, les acceptions de ces doublets se sont différenciées. C'est ainsi que l'hébreu contemporain utilisera le mot kayits pour désigner l'été, la saison couverte par juin-juillet-août, tandis que le doublet d'origine araméenne kayit, décrit le repos estival, les vacances d'été (et l'on commence même à parler de kayit pour désigner la détente et la villégiature d'hiver). Pour prendre un autre exemple, l'hébreu aura recours au verbe hébraïque qui signifie «sortir»: latsète (et à la forme factitive : «faire sortir»/lehotsi) dans le vocabulaire général. Mais il a recours au doublet araméen nefak pour des emplois spécialisés : émettre des timbres, des billets, des médailles, frapper de la monnaie (lehanepik, au sens anglais de to issue).

\subsection{La dépendance de l'Académie vis-à-vis d'autres institutions}

L'Académie de la langue hébraïque est l'instance législative qui fixe l'usage normatif. Mais comme tout pouvoir législatif, elle dépend d'institutions exécutives. Il s'agit essentiellement du ministère de l'Éducation et de la Culture ainsi que des médias : presse électronique et écrite. Dans ses rapports avec le ministère, il faut déplorer que les décisions de l'Académie ne soient pas toujours mises à exécution. Et lorsqu'elles le sont, c'est avec un grand retard. C'est ainsi que les décisions concernant l'orthographe attendent toujours d'être appliquées. Le poids de la routine, chez les fonctionnaires, et la crainte de soulever l'opposition du public contre des changements d'habitude dissuadent les responsables de mettre en œuvre les décisions de l'Académie. On se réjouira cependant que la Division des programmes d'étude, auprès du ministère de l'Éducation et de la Culture, fait beaucoup pour enraciner l'hébreu normatif. En ce qui concerne les médias, la radio et la télévision d'État emploient des conseillers linguistiques. Il s'agit d'employés de l'Académie soigneusement sélectionnés par elle. Le résultat est que les programmes en différé, donc après montage, respectent soigneusement les normes du bon usage. C'est encore plus vrai pour les textes lus par des speakers, notamment les bulletins d'information. Bien plus, nombre de néologismes forgés par l'Académie sont immédiatement intégrés dans le langage courant grâce à ce lien, formel et institutionnalisé, entre l'Académie et l'Office de radiodiffusion et télévision israélien (l'O.R.T.I.). Néanmoins, il va sans dire que dans les reportages, les débats et autres émissions «spontanées», il n'y a pas (et il ne peut y avoir) de contrôle de la part d'un conseiller linguistique. Tout dépend donc du locuteur, de son niveau d'instruction et de ses connaissances linguistiques. Le vrai problème concerne plutôt le statut de la langue radiodiffusée dans les chaînes de radio et de télévision créées récemment, notamment celles sur lesquelles l'État n'exerce aucun contrôle. À dire vrai, elles ont commencé à introduire d'elles-mêmes des réviseurs. Quant aux journaux, la plupart n'emploient pas de réviseurs, mais il faut reconnaître que beaucoup de rédacteurs en chef veillent soigneusement à la qualité de la langue imprimée et n'hésitent pas à consulter l'Académie. N'oublions pas que l'Académie possède ses propres publications qui lui permettent d'établir un contact direct avec le public. Ne nous leurrons pas, il s'agit d'un public restreint, plutôt que du grand public. Mais ces publications méritent qu'on s'y arrête. 


\subsection{Les publications de l'Académie}

En dehors des publications scientifiques de l'Académie, dont nous ne parlerons pas ici, il existe une série d'organes qui permettent de porter à la connaissance du public les décisions de l'Académie. Mentionnons tout d'abord les dizaines de glossaires et dictionnaires spécialisés dans tous les domaines. (La plupart sont bilingues : anglais-hébreu, hébreu-anglais.) Nous venons de publier successivement un Dictionnaire de dentisterie (que possèdent aujourd'hui de nombreux dentistes israéliens), la première partie d'un Dictionnaire de termes bancaires et du marché des capitaux (1996), déjà acheté par des centaines de spécialistes, et un Dictionnaire de l'intelligence artificielle (1997). D'autres ouvrages sont en préparation dont un Dictionnaire de la diplomatie, pratiquement achevé. L'Académie diffuse également une publication trimestrielle : Lechonènou laam («Notre langue pour le peuple»). Ce journal, écrit dans un langage accessible à tous, traite de questions de bon usage. On y trouve également des questions d'intérêt général que se pose le public en matière de langue, ainsi que les réponses apportées par le secrétariat scientifique de l'Académie.

Six fois par an, l'Académie édite également une affichette, au graphisme particulièrement soigné, qui présente quelques néologismes et corrige des fautes courantes, intitulée : Lamed ète lechonekha («Enseigne ta langue»). Près de deux cents de ces affichettes ont déjà été publiées et elles jouissent d'une telle popularité que l'Académie les a reliées et a publié, en 1994, les 180 premières fiches dans un volume muni d'index détaillés. Le livre a été réédité en 1995, à la demande du public.

Et puis, l'Académie publie les procès-verbaux de ses séances plénières. Depuis sa fondation et jusqu'à l'été 1993, près de quarante volumes ont vu le jour, contenant l'intégralité des débats et des résolutions de l'Académie, ainsi que divers exposés faits en plénière. À la radio publique, les conseillers linguistiques tiennent une rubrique quotidienne intitulée Règa chèl 'ivrit («La minute d'hébreu»). Cette rubrique est diffusée deux fois par jour à des heures de grande écoute. Une émission similaire (Higuiya zemane lachone, «c'est l'heure de la langue») est diffusée, une fois par semaine, à la télévision. Elle est présentée par un spécialiste très populaire en Israël, qui assure également une émission de radio quotidienne sur une autre chaîne. Soulignons que, sans entretenir de liens formels avec l'Académie, des dizaines d'institutions en Israël emploient des conseillers linguistiques qui, eux, font largement appel à nous. À ce propos, il convient de noter que notre secrétariat académique propose au public une «ligne ouverte». Il s'agit d'un service téléphonique régulier et permanent qui permet à l'Académie de répondre chaque jour à une vingtaine d'appels, avec une moyenne mensuelle supérieure à cinq cents appels. Les correspondants sont des réviseurs, des enseignants, des officiers; intellectuels ou gens du peuple, ils appartiennent à toutes les couches de la population. Leurs questions portent sur l'orthographe, la grammaire et le vocabulaire. Parallèlement, nous recevons par écrit des centaines de demandes similaires. Le bulletin de l'Académie, Acadèm, est diffusé à des milliers d'exemplaires et touche un public varié. On y trouve des nouvelles de l'Académie et de ses membres, un rapport sur les néologismes et sur les publications de l'Académie, un tour d'horizon de l'activité d'un membre choisi de l'Académie, des documents, etc.

En 1994, l'Académie a créé un nouvel institut de recherche, l'Institut Masie, consacré à l'étude de la renaissance linguistique hébraïque. En dehors de ses activités de recherche, il organise des journées d'étude pour les élèves des écoles et des lycées, les élèves d'oulpanim (centres d'apprentissage accéléré de l'hébreu, pour les immigrants) et pour le grand public. 


\section{EN GUISE DE CONCLUSION}

Malgré tous nos efforts, les publications, les programmes divers, le lien avec le public, il faut reconnaître, hélas, que trop souvent la qualité de la langue est le cadet des soucis de bien des Israéliens, y compris d'une partie des représentants du pouvoir, qui donnent le mauvais exemple en cette matière. Mais l'Académie sait bien qu'elle doit poursuivre ses efforts. Pour parvenir à inculquer une langue de culture au sein de toutes les couches de la société, il lui faut obtenir le plein concours du ministère de l'Éducation, véritable responsable de l'enseignement de l'hébreu. L'Académie est consciente de l'importance de l'influence qu'elle doit continuer d'exercer sur les médias. Mais pour réussir, il faut du temps et de la patience. La renaissance de l'hébreu a constitué un véritable miracle sans précédent dans l'histoire du monde et de la linguistique. Elle est la preuve de ce que l'on peut réaliser avec de la détermination. De fait, comme dans toute entreprise d'envergure qui a réussi, on a tendance à considérer le résultat comme allant de soi et à faire preuve d'une certaine nonchalance, à croire qu'il n'est plus besoin de se battre et de lutter pour conserver les acquis. Il en va ainsi pour la langue hébraïque. À force d'efforts, l'hébreu est devenu une langue bien vivante et dynamique. Certains ont tendance à oublier qu'il doit être préservé, défendu contre un développement anarchique. Les responsables culturels et leurs porte-parole (l'Académie en tête) se doivent de fournir les réponses appropriées pour endiguer de telles attitudes.

Traduit et adapté de l'hébreu par Francine Kaufmann. 